\title{
Outcomes of three-Tesla magnetic resonance imaging for the identification of pituitary adenoma in patients with Cushing's disease
}

\author{
Noriaki Fukuhara ${ }^{1), 2), 3)}$, Naoko Inoshita ${ }^{2), 3), 4)}$, Mitsuo Yamaguchi-Okada ${ }^{1)}$, Keita Tatsushima ${ }^{5}$, \\ Akira Takeshita $^{3), 5)}$, Junko Ito ${ }^{\text {(), Yasuhiro Takeuchi }}{ }^{3), 5)}$, Shozo Yamada ${ }^{3), 7)}$ and Hiroshi Nishioka ${ }^{1), 3)}$ \\ 1) Department of Hypothalamic and Pituitary Surgery, Toranomon Hospital, Tokyo 105-8470, Japan \\ 2) Department of Comprehensive Pathology, Graduate School of Medical and Dental Sciences, Tokyo Medical and Dental University, \\ Tokyo 113-8510, Japan \\ 3) Okinaka Memorial Institute for Medical Research, Tokyo 105-8470, Japan \\ 4) Department of Pathology, Toranomon Hospital, Tokyo 105-8470, Japan \\ 5) Department of Endocrinology and Metabolism, Toranomon Hospital, Tokyo 105-8470, Japan \\ 6) Department of Pediatrics, Toranomon Hospital, Tokyo 105-8470, Japan \\ 7) Department of Neurosurgery, Tokyo Neurological Center Hospital, Tokyo 134-0088, Japan
}

\begin{abstract}
Pituitary adenoma has been reported to be detectable in only 36-63\% of Cushing's disease (CD) patients by magnetic resonance imaging (MRI). In this study, we investigate the outcomes and problems associated with tumor identification using 3-Tesla (3-T) MRI, which provides clearer images than $\leq 1.5$-T MRI, in 115 patients who were initially diagnosed with $\mathrm{CD}$. Before surgery, 31 macroadenomas (27\%) and 54 microadenomas (47\%) were identified by 3-T MRI, but pituitary adenoma was invisible on MRI in the remaining 30 cases $(26 \%)$. The smallest tumor diameter amenable to a definitive diagnosis was $2 \mathrm{~mm}$, and spoiled gradient-echo was the best sequence for diagnosing microadenomas. In 14 of 30 cases of MRI-invisible CD, the pituitary adenoma was identified during surgery. Nine of these 14 tumors that developed from outside the pituitary gland were retrospectively identified on MRI by comparison with surgical findings. The remaining 16 cases of MRI-invisible $\mathrm{CD}$ in which the pituitary adenoma was not identified during surgery involved partial hypophysectomy. Seven cases were hormonally remitted, but another nine cases experienced persistent disease after surgery. The sensitivity and specificity of the pituitary adenoma diagnosis in CD patients after the introduction of 3-T MRI were $80 \%$ and $100 \%$, respectively. However, the sensitivity decreased to $72 \%$ when macroadenomas were excluded. Some adenomas associated with CD are still undetectable on 3-T MRI due to tumor size, location and intensity. However, sensitivity can be improved by monitoring tumors that develop outside the pituitary gland.
\end{abstract}

Key words: Cushing's disease, Magnetic resonance imaging, Inferior petrosal sinus sampling, Ectopic pituitary adenoma, Exophytic pituitary adenoma

IN CUSHING'S DISEASE (CD), pituitary adenomas are detectable in only $36-63 \%$ of cases by magnetic resonance imaging (MRI) [1]. Venous sinus sampling is required for tumor-invisible $\mathrm{CD}$ by MRI, although this test is invasive. Inferior petrosal sinus sampling (IPSS) was reported to be the gold standard for the diagnosis of CD because it achieved $81-100 \%$ sensitivity with CRH stimulation and was superior to pituitary MRI [2]. However, we reported that the sensitivity of IPSS for tumor-

Submitted Oct. 25, 2018; Accepted Jan. 3, 2019 as EJ18-0458 Released online in J-STAGE as advance publication Feb. 14, 2019 Correspondence to: Naoko Inoshita, Department of Pathology, Toranomon Hospital, 2-2-2 Toranomon, Minato-ku, Tokyo 105-8470, Japan.

E-mail: inonao3939@gmail.com invisible CD by MRI was only $50 \%$ (100\% specificity) [3]. The poor performance of MRI and the lack of useful sequences may be responsible for the lower sensitivity of the CD tumor diagnosis on MRI and the better outcome of IPSS. The use of high-magnetic field MRI and various sequences may improve the sensitivity of tumor detection in $\mathrm{CD}$ patients, resulting in a lower sensitivity of IPSS for tumor-invisible CD. Recently, 3-Tesla (3-T) MRI, which provides clearer images than $\leq 1.5-\mathrm{T}$ MRI, has become more widespread. In this study, we investigated whether the diagnosis of pituitary adenoma in CD patients was improved after the introduction of 3-T MRI and discussed its limitations. 


\section{Methods}

\section{Study design}

This study is the retrospective case series study and was approved by the Institutional Review Board of Toranomon Hospital (No. 1616). We investigated outcomes and problems in tumor identification by MRI for endocrinologically diagnosed $\mathrm{CD}$ after introduction of 3-T MRI. Three-T MRI was introduced at Toranomon Hospital in August 2012.

\section{Patients}

Between August 2012 and May 2017, 115 patients underwent initial surgery for endocrinologically diagnosed CD at Toranomon Hospital. These 115 patients were included in this study. CD patients who received prior surgery were excluded due to the possibility of bias in their MRI diagnosis. Patients who underwent 3-T MRI were included in part of our previous report from 2012, but these patients received 3-T MRI at other hospitals. The patients in this study did not overlap with the cases included in our 2012 report [3], although part of this population overlapped with the cases from our 2015 report [4].

\section{MRI}

MRI was performed using a 1.5-T MRI (1.5T MAGNETOM Symphony, Siemens Healthcare, Erlangen, Germany, and 1.5-T Excelart Vantage Power Plus, Toshiba Medical Systems, Tokyo, Japan) or a 3.0-T MRI (3.0T Ingenia, Philips Healthcare, Best, The Netherlands) system. As routine sequences, coronal and sagittal sections were obtained using nonenhanced T1-weighted spin echo (SE), T2-weighted SE and enhanced T1-weighted SE sequences. Some patients with macroadenoma or microadenoma were not performed 3-T MRI because their tumors were still identified on 1.5-T MRI. All cases with microadenomas or tumors invisible on MRI were also imaged with a 3D-spoiled gradient-echo sequence (SGE). A dynamic study was performed with a limited number of cases. If MRI performed at former hospitals where the patients were referred from were available, MRI of former hospitals were compared with our 3-T MRI. However, systems and magnetic fields of MRI of former hospitals were not unknown.

\section{IPSS}

IPSS was performed for patients whose MRI examination did not reveal the pituitary adenoma required to diagnose CD. IPSS was performed with catheterization of the bilateral inferior petrosal sinus (IPS), and blood samples were collected before and $0,3,5$, and 10 minutes after $\mathrm{CRH}$ administration. An IPS/peripheral
ACTH ratio $\geq 2$ before $\mathrm{CRH}$ administration or a peak ratio $\geq 3$ after $\mathrm{CRH}$ administration was considered indicative of CD. If the IPSS result did not satisfy either of these criteria, then the IPS/peripheral ACTH ratio was normalized with the IPS/peripheral PRL ratio. CD was also diagnosed if the normalized central/peripheral ACTH ratio was $\geq 1.3$ [5].

\section{Results}

MRI identified a pituitary adenoma in 85 cases $(74 \%)$ before surgery. The remaining 30 cases $(26 \%)$ were not diagnosed with a pituitary adenoma before surgery. Of the 85 cases in which pituitary adenomas were preoperatively identified by MRI, 31 cases $(27 \%)$ were macroadenomas $(\geq 10 \mathrm{~mm})$, and 54 cases $(47 \%)$ were microadenomas $(<10 \mathrm{~mm})$. Two of the 54 cases of microadenomas were diagnosed on the 1.5-T MRI system; therefore, they did not undergo additional 3-T MRI. In 30 cases of tumor-invisible $\mathrm{CD}$, the pituitary adenoma was identified during surgery and was hormonally remitted after surgery in 14 cases. In the 16 cases in which the pituitary adenoma was not identified during surgery, seven cases were hormonally remitted after partial hypophysectomy, but another nine cases showed persistent disease despite partial hypophysectomy. These results indicates that sensitivity and specificity of pituitary adenoma identification in CD patients by MRI was $80 \%$ $(85 / 106)$ and $100 \%(9 / 9)$. However, the sensitivity decreased to $72 \%(54 / 75)$ when a macroadenoma was excluded.

\section{Microadenomas}

In the 54 cases in which microadenomas were preoperatively identified on MRI, the minimum tumor diameter was $2 \mathrm{~mm}$. The sensitivity of tumor identification with each sequence was as follows: SGE, 100\% (54/54); enhanced T1-weighted SE, 93\% (50/54); and T2weighted SE, 63\% (34/54). These results indicated that SGE was the best sequence for identifying microadenomas in CD patients. The maximum tumor diameter of the pituitary adenomas that were identified by the SGE sequence but not by the enhanced T1-weighted SE sequence was $5 \mathrm{~mm}$, whereas the maximum diameter of those that were not identified by the T2-weighted SE sequence was $7 \mathrm{~mm}$. These 54 cases of microadenomas included three cases of exophytic pituitary adenomas that developed from the lateral surface of the pituitary gland rather than inside the gland.

The results from MRI performed at former hospitals where the patients were referred from were available for 49 of the 54 cases with a microadenoma. Although 15 of the 49 cases $(31 \%)$ were not diagnosed with a pituitary 
adenoma causing $\mathrm{CD}$, the tumor was eventually diagnosed by 3-T MRI. In particular, two of these 15 patients received long-term medical therapy because the cause of CD was not diagnosed by $1.5-\mathrm{T}$ MRI. The tumor was identified retrospectively on MRI performed at former hospitals in eight of the 15 cases after the tumor was diagnosed with 3-T MRI. However, the tumor was not diagnosed at former hospitals because the tumor was indistinct on MRI performed at that hospital. On the other hand, seven of the 15 cases were newly diagnosed with 3-T MRI in our hospital because tumors were not identified on MRI performed at former hospitals.

\section{Tumor-invisible CD}

All 30 cases with tumors that were not preoperatively identified by MRI were performed 3-T MRI and IPSS was performed for 28 of 30 . All but one case showed a positive IPSS result; the result of the one exception was controversial because this case showed periodic Cushing syndrome that was in a remission period when IPSS was performed. IPSS was difficult to perform in two cases because one patient was a pediatric patient, and another could not discontinue metyrapone therapy due to Pneumocystis pneumonia. These patients received pituitary surgery after CD was diagnosed by several hormonal tests other than IPSS.

The pituitary adenomas of nine of the 14 cases that were not preoperatively diagnosed by MRI were intraoperatively identified as attached to the lateral surface of the normal pituitary gland. These nine adenomas were retrospectively identified on preoperative 3-T MRI by comparison with the surgical findings. However, the lesions were not suspected to be pituitary adenomas because they did not derive from the inner part of the pituitary gland (Fig. 1, upper). One of these nine cases was especially difficult to diagnose on MRI preoperatively because the tumor was very small $(1 \mathrm{~mm}$ in diameter; Fig. 1, middle). Two of the 14 cases were intracavernous sinus ectopic pituitary adenomas. These tumors were not diagnosed preoperatively on 3-T MRI but were retrospectively identified (Fig. 1, lower). In the remaining three of these 14 cases, a pituitary adenoma with a diameter of 3-5 mm was identified during surgery, but the intensity of these adenomas was equal to that of the normal pituitary gland on MRI. Therefore, the pituitary adenomas could not be identified by MRI even when retrospectively compared to the surgical findings.

In six of seven cases of tumor-invisible CD that were cured after partial hypophysectomy, the pituitary adenoma was identified on the pathological specimen. These cases were not diagnosed with a pituitary adenoma on 3-T MRI because the tumor was extremely small. Nine cases with tumor-invisible $\mathrm{CD}$ that did not exhibit a change in hormone status despite partial hypophysectomy were not identified as ectopic ACTH-secreting tumors by systemic medical examination. One patient of nine cases who were not cured after surgery, showed negative IPSS result because this patient showed periodic Cushing's syndrome. Another patient did not underwent IPSS because this patient could not discontinue metyrapone therapy.

A dynamic T1-weighted contrast-enhanced sequence was performed in 11 of the 30 cases with tumors that were not identified by other sequences on 3-T MRI. In these 11 cases, the pituitary adenoma was retrospectively identified in only two cases in which the pituitary adenoma was retrospectively identified after surgery. However, the tumor was not diagnosed before surgery. In another nine cases (six cured after surgery and three not cured) in which the pituitary adenoma was not identified on 3-T MRI even when compared to the surgical findings, the pituitary adenoma was also not identified by dynamic MRI.

These results are summarized in the flow chart in Fig. 2.

\section{Complicating incidentaloma}

A complicating Rathke cleft cyst (RCC) was identified in six $(7 \%)$ of 84 cases with CD excluding macroadenomas. Of these six cases, two cases were microadenomas and four cases were tumor-invisible CD. These six RCCs were correctly diagnosed as RCCs before surgery in our hospital, although RCC was diagnosed as the responsible pituitary adenoma in two of the six cases in former hospitals. In this study, no complicating pituitary incidentaloma responsible for CD was found.

\section{Discussion}

\section{Overall outcomes}

Our results indicated that the use of 3-T MRI for the diagnosis of pituitary adenomas in CD patients showed $80 \%(85 / 106)$ sensitivity and 100\% (9/9) specificity. However, the sensitivity decreased to $72 \%$ (54/75) when macroadenomas were excluded. The best sequence for identifying the pituitary adenoma in CD was SGE. This result supports recent reports that $\mathrm{SGE}$ is superior to dynamic contrast T1-weighted SE [6, 7]. The reasons why the pituitary adenomas in the CD patients were not identified were associated with tumor location, tumor size, and the MRI intensity of the tumor.

\section{Diagnosis of microadenomas}

Fifteen of $49(31 \%)$ microadenomas that were not identified in other hospitals were newly diagnosed using 3-T MRI, indicating that 3-T MRI can improve the sensitivity of tumor identification in CD patients. Part of the 


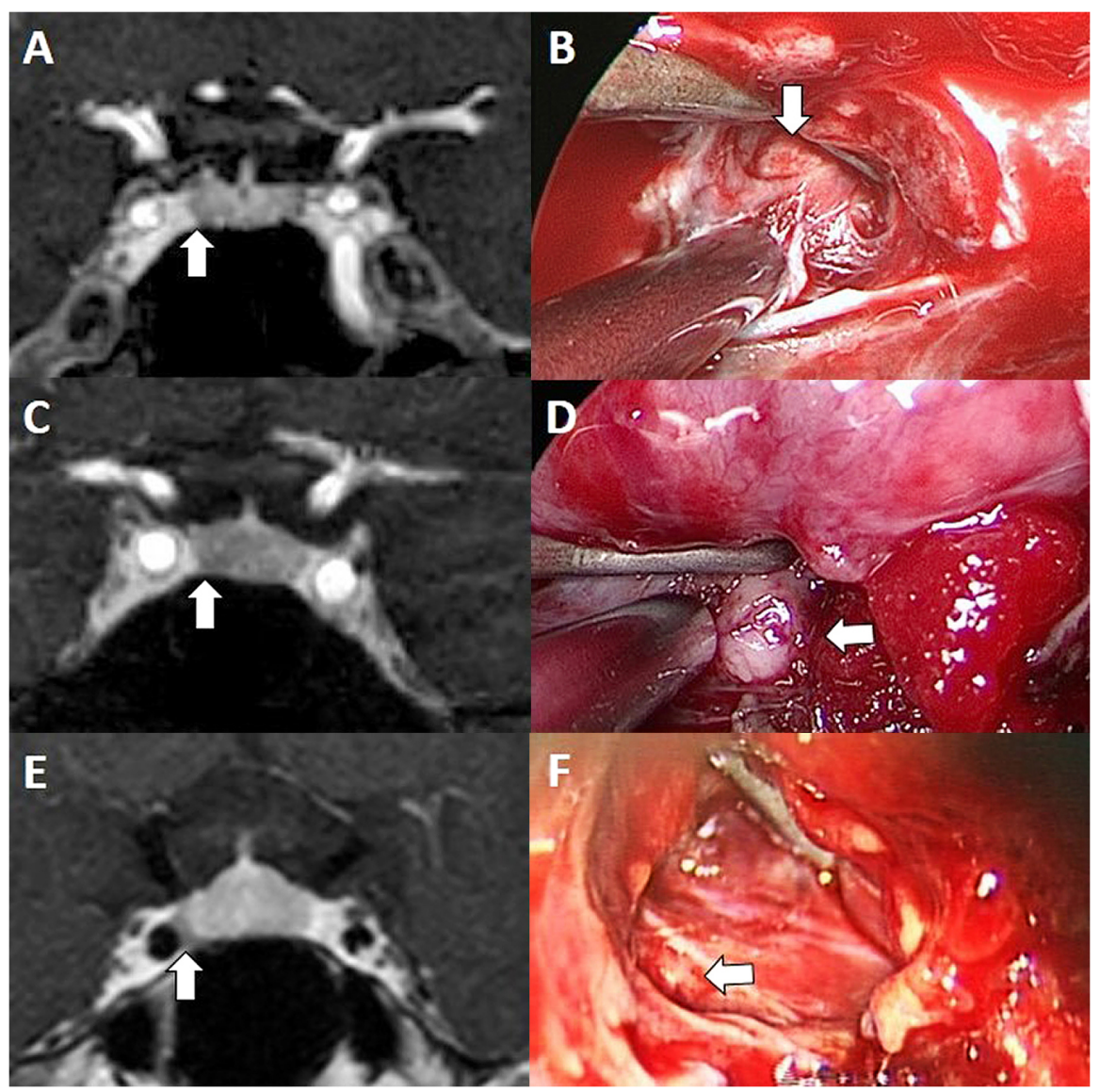

Fig. 1 MRI and surgical findings of exophytic pituitary adenomas that developed from the lateral surface of the normal pituitary gland and an intracavernous sinus ectopic pituitary adenoma.

A: MRI of a microadenoma (arrow) attached to the right side of the pituitary gland. B: Intraoperative findings related to A (arrow indicates the tumor). C: MRI of a 1-mm microadenoma (arrow). D: Intraoperative findings related to C (arrow indicates the tumor). E: MRI of an ectopic pituitary adenoma (arrow) occurring in the right cavernous sinus. F: Intraoperative findings related to $\mathrm{E}$ (arrow indicates the tumor inside the cavernous sinus).

tumor was retrospectively identified on MRI at the former hospital after identification on 3-T MRI; however, the tumors may not have been diagnosed in former hospitals because the images were more obscure than the 3-T MR images. Although the comparison between our 3-T MRI results and the MRI results obtained in former hospitals in this study was not strict due to differences between the systems, methods and experience of the physicians, many tumors were newly diagnosed by $3-\mathrm{T}$ MRI. One study reported that the sensitivity for identifying pituitary tumors on MRI improved from 54\% to $85 \%$ with 3-T MRI compared to 1.5-T MRI [8]. Therefore, 3-T MRI may be superior to 1.5-T MRI for identification of pituitary microadenomas in $\mathrm{CD}$ patients.

\section{Exophytic and ectopic pituitary adenomas}

Some tumors in CD patients that were not preoperatively identified could be retrospectively identified by referring to the surgical findings. These tumors were exophytic pituitary adenomas that developed from the lateral surface of the pituitary gland or ectopic pituitary adenomas that developed in the cavernous sinus. Since pituitary adenomas usually develop inside the pituitary gland, identifying these tumors outside the pituitary gland as pituitary adenomas before surgery is difficult. However, we could retrospectively identify these tumors by comparing the intraoperative findings with the MRI findings. The reason why these exophytic or ectopic pituitary adenomas were not identified before surgery was not due to MRI performance but rather the physician's ability. These exophytic or ectopic pituitary adenomas were not rare because they were identified in $10 \%$ (12 of 115 cases) of all CD patients who underwent initial surgery in this study. In addition, three of the 10 exophytic pituitary adenomas could be preoperatively identified, indicating that identifying exophytic or ectopic pituitary adenomas on MRI before surgery is not impossible. The sensitivity of preoperative tumor diag- 


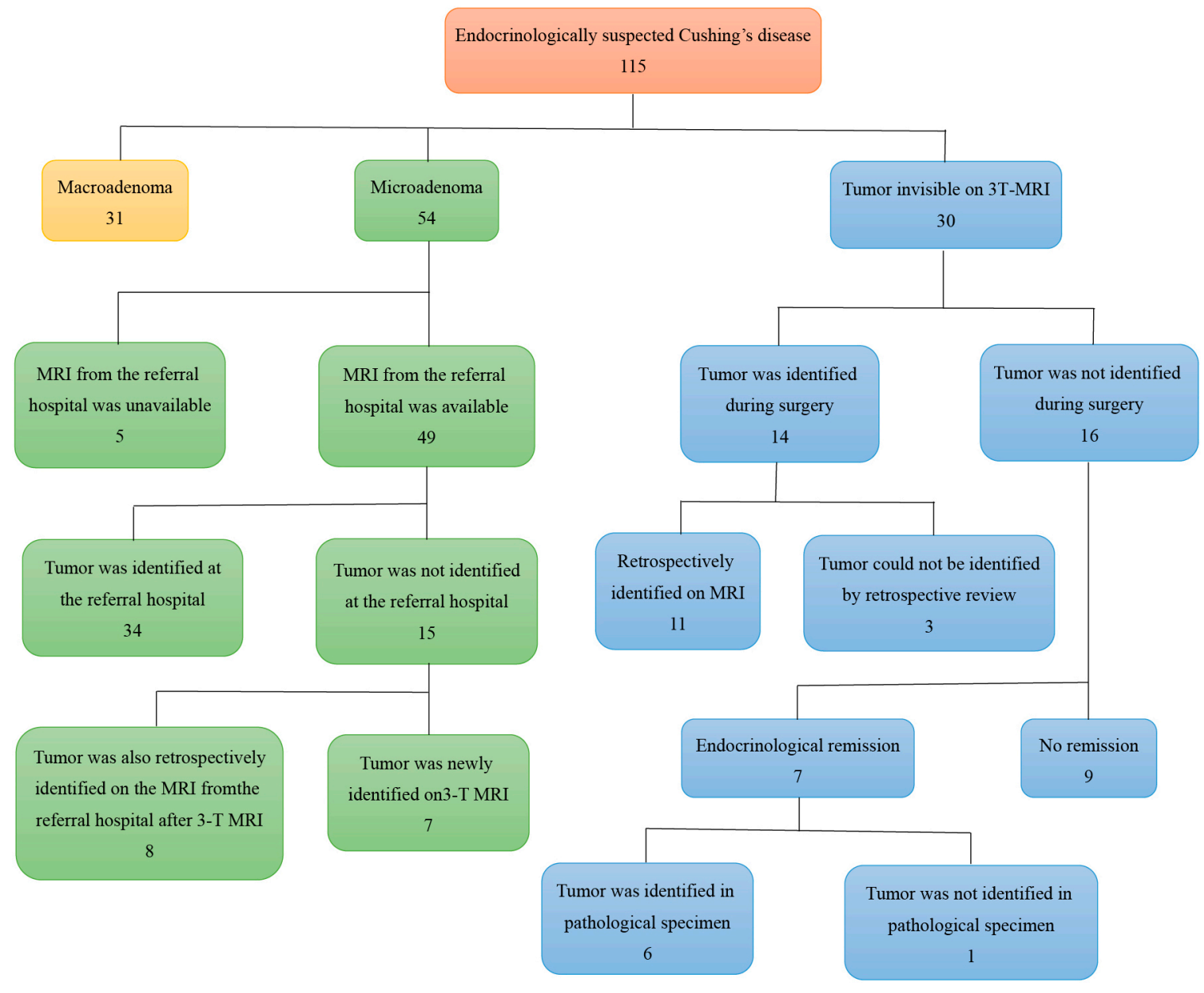

Fig. 2 Summary of 115 cases of Cushing's disease

nosis on MRI may be improved by focusing on these exophytic or ectopic pituitary adenomas.

\section{Limitations of the detectable tumor size}

Although one exophytic pituitary adenoma with a diameter of $1 \mathrm{~mm}$ was retrospectively identified by $3-\mathrm{T}$ MRI after comparison with the surgical findings, identification before surgery was considered difficult due to the possibility of a false-positive [9]. Therefore, the minimum tumor diameter that could be identified before surgery was $2 \mathrm{~mm}$, even with 3-T MRI. Similarly, tumors with a diameter of 3-8 $\mathrm{mm}$ were reported to be detectable using the SGE sequence [7]. However, three microadenomas with 3-5-mm diameters were not identified by 3-T MRI because their intensity on enhanced T1weighted images was almost the same as that of the normal pituitary gland. Not all microadenomas with a diameter $>2 \mathrm{~mm}$ can be identified on 3-T MRI. Moreover, seven of 30 cases of tumor-invisible CD were endocrinologically cured after partial hypophysectomy, and six of these seven pituitary adenomas were diagnosed in pathological specimens. One remaining prob- lem is very small pituitary adenomas that are impossible to identify using 3-T MRI. In the future, higher magnetic field MRI may resolve this problem [10].

\section{Complicating incidentaloma}

Pituitary incidentalomas have been identified on MRI in $10-38 \%$ of the general population, and RCC has been found in 13-33\% of autopsy cases [11-13]. RCC can complicate pituitary adenomas [14]. However, RCC can be misdiagnosed as the tumor responsible for $\mathrm{CD}$ when the pituitary adenoma is not clearly identified on MRI. In this study, RCC was diagnosed based on characteristic findings on MRI. However, the possibility of an incidentaloma or RCC must be considered when diagnosing CD based on imaging examinations.

\section{Conclusions}

The sensitivity and specificity of the diagnosis of pituitary adenoma in $\mathrm{CD}$ patients after the introduction of 3-T MRI were $80 \%$ and $100 \%$, respectively, but the sensitivity decreased to $72 \%$ when macroadenomas were 
excluded. Although some tumors could not be identified using 3-T MRI, several microadenomas were newly identified by 3-T MRI. SGE was the best sequence for diagnosing microadenomas in $\mathrm{CD}$. The smallest tumor diameter amenable to definitive diagnosis was $2 \mathrm{~mm}$. The reasons that tumors were not identified even on 3-T MRI were tumor size, location and intensity on MRI in addition to the physician's skill. The sensitivity of this approach may be improved by focusing on tumors that develop on the lateral surface of the pituitary gland or ectopic pituitary adenomas.

\section{Disclosure}

None of the authors has any potential conflicts of interest associated with this research.

\section{References}

1. Ludecke DK, Flitsch J, Knappe UJ, Saeger W (2001) Cushing's disease: a surgical view. J Neurooncol 54: 151166.

2. Pecori Giraldi F, Cavallo LM, Tortora F, Pivonello R, Colao A, et al. (2015) The role of inferior petrosal sinus sampling in ACTH-dependent Cushing's syndrome: review and joint opinion statement by members of the Italian Society for Endocrinology, Italian Society for Neurosurgery, and Italian Society for Neuroradiology. Neurosurg Focus 38: E5.

3. Yamada S, Fukuhara N, Nishioka H, Takeshita A, Inoshita $\mathrm{N}$, et al. (2012) Surgical management and outcomes in patients with Cushing disease with negative pituitary magnetic resonance imaging. World Neurosurg 77: 525-532.

4. Yamada S, Inoshita N, Fukuhara N, Yamaguchi-Okada M, Nishioka H, et al. (2015) Therapeutic outcomes in patients undergoing surgery after diagnosis of Cushing's disease: a single-center study. Endocr J 62: 1115-1125.

5. Sharma ST, Raff H, Nieman LK (2011) Prolactin as a marker of successful catheterization during IPSS in patients with ACTH-dependent Cushing's syndrome. $J$ Clin Endocrinol Metab 96: 3687-3694.

6. Grober Y, Grober H, Wintermark M, Jane JA Jr, Oldfield EH (2018) Comparison of MRI techniques for detecting microadenomas in Cushing's disease. J Neurosurg 128: 1051-1057.

7. Kasaliwal R, Sankhe SS, Lila AR, Budyal SR, Jagtap VS, et al. (2013) Volume interpolated 3D-spoiled gradient echo sequence is better than dynamic contrast spin echo sequence for MRI detection of corticotropin secreting pituitary microadenomas. Clin Endocrinol (Oxf) 78: 825830.

8. Vitale G, Tortora F, Baldelli R, Cocchiara F, Paragliola $\mathrm{RM}$, et al. (2017) Pituitary magnetic resonance imaging in Cushing's disease. Endocrine 55: 691-696.

9. Batista D, Courkoutsakis NA, Oldfield EH, Griffin KJ, Keil M, et al. (2005) Detection of adrenocorticotropinsecreting pituitary adenomas by magnetic resonance imaging in children and adolescents with cushing disease. $J$ Clin Endocrinol Metab 90: 5134-5140.

10. de Rotte AA, Groenewegen A, Rutgers DR, Witkamp T, Zelissen PM, et al. (2016) High resolution pituitary gland MRI at 7.0 tesla: a clinical evaluation in Cushing's disease. Eur Radiol 26: 271-277.

11. Freda PU, Beckers AM, Katznelson L, Molitch ME, Montori VM, et al. (2011) Pituitary incidentaloma: an endocrine society clinical practice guideline. J Clin Endocrinol Metab 96: 894-904.

12. Osborn AG, Preece MT (2006) Intracranial cysts: radiologic-pathologic correlation and imaging approach. Radiology 239: 650-664.

13. Teramoto A, Hirakawa K, Sanno N, Osamura Y (1994) Incidental pituitary lesions in 1,000 unselected autopsy specimens. Radiology 193: 161-164.

14. Karavitaki N, Scheithauer BW, Watt J, Ansorge O, Moschopoulos M, et al. (2008) Collision lesions of the sella: co-existence of craniopharyngioma with gonadotroph adenoma and of Rathke's cleft cyst with corticotroph adenoma. Pituitary 11: 317-323. 